\title{
Future Prospects of Health Informatics Summarized from the Special Issue 'Women in Health Informatics' to the Memory of Professor Jana Zvárová
}

\author{
Pirkko Nykänen* \\ Faculty of Natural Sciences, University of Tampere, Tampere, Finland
}

\begin{abstract}
This paper summarizes the future prospects of health informatics as seen by the authors of the Special Issue 'Women in Health Informatics'. The prospects are focused on health informatics important themes like patientcentered care and patient empowerment, data, secondary use of data, big data, security of data, Internet and emerging
\end{abstract}

Correspondence to:

\section{Pirkko Nykänen}

Faculty of Natural Sciences, University of Tampere

Tampere, Finland

E-mail: Pirkko.Nykanen@uta.fi technologies, electronic health records, health informatics education, evaluation, social aspects of health information systems and legal and standardization issues.

\section{Keywords}

Medical informatics; Health informatics; Healthcare informatics; eHealth; Data

\section{$1 \quad$ Introduction}

The Special Issue on Women in Health Informatics was initiated and planned with Professor Jana Zvárová. We three editors, Jana, Pirkko and Diane, worked intensively together in planning the issue, in inviting people to contribute and in reviewing their submissions. Jana was very happy and enthusiastic that we had been successful to invite so many distinguished colleagues to provide their stories and share their experiences on their career development in the health and biomedical informatics domain. We all felt that we had found with this Special Issue a valuable way to allow the voice of women and to highlight the achievements on women in the very challenging and multidisciplinary field of health informatics.

After the decease of Professor Jana Zvárová, it was very clear to us, Pirkko and Diane, that we continue, we finalize the work started. The Special Issue [1], published in November 2017, has two purposes: It is a memorial to Professor Zvárová, and her EUROMISE work, but also a tribute to the women, who have provided their career stories, all written in their own voices, more than a dozen exceptional women from around the globe, who have worked over the decades in bioinformatics, health informatics and digital health. Each paper also provides a view of the health informatics work that has been undertaken in their own country, problems
EJBI 2018; 14(2):46-48

Received: March 09, 2018

Accepted: March 21, 2018

Published: April 30, 2018 encountered and insights into where health informatics may be seen to be heading in the future.

In this paper, we summarize the major findings of future prospects of health informatics as presented by the women in their career stories $[2,3,4,5,6,7,8,9,10,11,12,13,14$, $15,16]$. These prospects reflect the various situations and backgrounds of women working as research scientists in this field. The prospects have been collected by walking-through all the women's articles and by condensing the findings. These prospects also devise the visions that are paving the way to the future of health informatics.

\section{Future of Health Informatics - Major Issues Raised by Professional Women}

Future will be the patient-centered; healthcare will be a process that crosses institutional and professional boundaries and cross-institutional eHealth architectures and infrastructures will continue to grow and support real patient-centered care and patient empowerment. Medical informatics has focused much by far on how to support doctors, nurses and other health care professionals in their work. But ultimately, it is the patient who has to decide what to do next. It is recognized that one of the key approaches to managing the spiraling costs of healthcare and of improving 
outcomes is to empower citizens, who after all represent the greatest single underutilized resource, and encourage them to take responsibility for managing their own healthcare. This involves moving away from healthcare systems which are focused primarily on treating illness to ones which are focused on promoting health and well-being. Already today, patients are better informed than ever before, and they have demands for valid and trusted online health information which in turn means improvements in quality standardization and accessibility of tools like the HONcode.

Another important issue in the future and, already today, is the data. The current trends with all available data are: Clinical research is going towards personalized and precision medicine, health care providers are eager to measure the effect of their actions and to build a learning health care system, and data science is the new buzzword in computer science albeit not clearly distinguishable from conventional statistical methods for data mining. We need methods, tools and infrastructures to support data-driven medical care and biomedical research. The future health and wellness information systems need to take not only the biomedical and clinical data of a patient into account but also patient generated data on daily activities, patient preferences, and environmental context for an individualized decision that really meets the patients' expectations, values and needs. Currently, these soft factors for medical decision support are not sufficiently understood and researched in all dimensions. Data access, availability and shareable use should not be seen as a threat to professional practice or data-subject preferences, but should be handled sensitively and openly. Informed use will ultimately be beneficial and holistic records, right-sourced and evidence-based, will support population well-being.

With data an important issue is the secondary use of clinical data to support research and health care practice. There is so much patient data available now in an electronic format that offer tremendous opportunities for precision medicine, decision support, or new scientific insights. Also reuse of routinely collected data, e.g. for calculating quality indicators, is needed to obtain and provide new knowledge without additional data collection. To be able to use all the available data from electronic health record, legal regulation is necessary and more research is needed on natural language processing, terminological systems and information models that are essential to standardize and structure routinely collected data.

The current hot topics in health informatics, such as big data, self-management of health and personalized or precision medicine need to be integrated with clinical research and large collaborative research projects together with medical departments and computer science departments. The technical infrastructures and processes, as well as Internet, Internet of Things (IoT) and social media provide huge volumes of interinstitutional and personal health and wellness data. The future is in the data.

We also need to concentrate on the use of enabling technologies in patient safety, process engineering, change management, human factors, stress management, and clinical point of care initiatives such as adherence and emphasis on patient-centric care. With the current enabling technologies and more recent analytic tools such as telemedicine, visualization, robotics, and machine learning that have become available, the field of health informatics can expect exponential growth in the use of technology to transform healthcare for the citizens of the world. The evolution of electronic health records and connectivity has also led to increased efforts to realize a vision of a data-driven national learning healthcare system to improve clinical knowledge and practice.

Theory-based development and evaluation of interventions are needed to understand the mechanisms behind these systems and to contribute to identifying the active ingredients of successful interventions. To obtain explicit evidence we need to perform more scientifically rigorous evaluation studies that show that the systems developed and used are acceptable, safe and effective and support health professionals in their clinical tasks as well as patients and citizens in managing their health and wellness. We also need to pay more attention to the design and implementation methods of health information systems in order to have predictably acceptable, safe and effective systems for user environments

All these future visions and developments with data and technologies emphasize that health informatics terminology should be standardized at the international level. We should achieve shared understandings on concepts, terms and processes to be able to use them in the same meaning in the global world. And, it is important to improve the legal practices, e.g. the medical informatics associations should insist on legal and ethical aspects of all activities dealing with information and communication technology in medicine and healthcare.

Medical informatics education is an important issue - it should be part of education of all the health professionals as the end-users of information technology in healthcare, and it should be properly located in their curriculum. New educational programs should support this, e.g. by offering part-time studies or online-based studies. Education should also cover more experts from non-technical backgrounds, such as a medical or nursing background, or with a background in quality management, risk management or process management, field of medical informatics. Medical informaticians should be able to act as a bridge between the two sides, but a little bit closer to medical, health care side - at the source of health information, with health professionals, and to understand their information needs.

\section{Summary and Conclusion}

All women $[2,3,4,5,6,7,8,9,10,11,12,13,14,15,16]$, who have provided their personal health informatics story to 
this special issue [1] have had the privilege to know Professor Zvárová, and to learn and to work with her. With these stories, each of us wants to pay tribute to, and remember, the achievements of Jana, as a professor, as a colleague and as a friend. These stories by qualified health informatics women also highlight Jana's influence on the health and bioinformatics as scientific disciplines and on the careers of all of us.

To summarize, the major challenge of medical informatics in the future will not only be to provide new methods for data analytics but to provide solutions that medical research results are available for use in patient care and for the patient itself. And, the health informatics community needs to find ways to build data models to systematically extract and link data along the continuum of care including their contexts and deliver the results according to research agendas that are driven by patient needs. These achievements lead to patient-centrality and a learning health system, where patients are involved in their health, are coached and motivated by clinical decision support, report outcome data, receive decision support, make shared decisions with their providers, and contribute to the body of evidence about treatment effectiveness, which could be mined by machine learning methods.

\section{Acknowledgements}

On behalf of all the women authors, I want to acknowledge Professor Jana Zvárová, for her initiative on this theme for the Special Issue, for her support, mentorship and collegiality throughout our careers, and her warm friendship. I also want to acknowledge Dr Diane Whitehouse, the other editor of the Special Issue, for excellent collaboration, both of us, Diane and me, want to acknowledge all these qualified women who have provided their stories, opened their career development and shared it with us all.

\section{References}

[1] Nykänen P, Whitehouse D. Women in health informatics, Special Issue. Int J Biomed Healthcare. 2017; 5.

[2] Ammenwerth E. An Austrian perspective on medical informatics. Int J Biomed Healthcare. 2017; 5: 4-8.
[3] Ball MJ. A personal journey into the field of healthcare informatics: looking back and considering the future. Int J Biomed Healthcare. 2017; 5: 9-15.

[4] Boyer C. e-Health in Switzerland: developments and challenges. Int J Biomed Healthcare. 2017; 5: 16-19.

[5] Grimson J. An Irish enhineer's perspective on health informatics. Int J Biomed Healthcare. 2017; 5: 20-22.

[6] de Keizer N. The health informatics research of a Dutch female scientist. Int J Biomed Healthcare. 2017; 5: 23-25.

[7] Kern J. Health or medical informatics in education, healthcare and research: The Croatian perspective. Int J Biomed Healthcare. 2017; 5: 26-29.

[8] Knaup P. The evolution of medical informatics in Germany: From structured clinical documentation to decision support for individualized therapy. Int J Biomed Healthcare. 2017; 5: $30-33$

[9] Koch S. Data collection health informatics in Sweden - a personal view. Int J Biomed Healthcare. 2017; 5: 34-37.

[10] Moen A. Diversity in health informatics. Int J Biomed Healthcare. 2017; 5: 38-40.

[11] Nykänen P. Health informatics - my way from medical technology to health informatics in Finland. Int J Biomed Healthcare. 2017; 5: 41-44.

[12] Peleg M. Health informatics in Israel; a focus on clinical decision support. Int J Biomed Healthcare. 2017; 5: 45-48.

[13] Roberts J. A long and varied career in International health informatics. Int J Biomed Healthcare. 2017; 5: 49-53.

[14] Seroussi B. From probability-bases systems to expert systems and guideline-based clinical decision support systems. Using health information technology to improve the quality of care. Int J Biomed Healthcare. 2017; 5: 54-57.

[15] Stoicu-Tivadar L. Healthcare informatics - a wonderland. Int J Biomed Healthcare. 2017; 5: 58-61.

[16] Whitehouse D. Digital health: reflections on an organic career. Int J Biomed Healthcare. 2017; 5: 62-65. 